Journal of

Accident and

Emergency

Medicine 1995

12, 208-209

\title{
Non-sustained broad-complex tachycardia following intravenous adenosine
}

\author{
A.D. HINGORANI \& T.H. FOLEY \\ Department of Medicine, Royal Surrey County Hospital, Guildford
}

\section{INTRODUCTION}

Adenosine induces short-lived atrioventricular nodal (AVN) blockade when injected intravenously and has superceded verapamil as the drug of choice for the termination of re-entrant paroxysmal supraventricular tachycardias (PSVT). ${ }^{1}$ It has also been found to be useful in the diagnosis of broad-complex tachycardia, distinguishing PSVT with aberrant conduction, which is terminated by adenosine, from preexcited atrial arrhythmias and ventricular tachycardia (VT), which are not. This agent's excellent safety profile is, in part, a reflection of its short half-life and the more frequent side-effects of dyspnoea, flushing and chest pain are transient and well tolerated. Although rhythm disturbances (predominantly bradyarrhythmias) have been described, few serious arrhythmia complications have been documented. We report the observation of non-sustained broadcomplex tachycardia following intravenous adenosine for the treatment of PSVT.

Keywords: adenosine, atrioventricular nodal blockade, broad complex tachcardia,. PSVT

\section{CASE HISTORY}

A 40-year-old female with a 20-year history of recurrent PSVT presented with a 3-h history of fast, regular palpitations of abrupt onset. Previous episodes had resolved spontaneously or with vagotonic manoeuvres, although adenosine $(12 \mathrm{mg}$ ) had been required on one occasion in the previous year. Medications on admission were sotalol (40 mg twice daily), L-thyroxine (250 mg once daily) for hypothyroidism following Hashimoto's thyroiditis and prednisolone (5 mg once daily) with hydroxychloroquine (200 mg twice daily) for systemic lupus erythematosus. Previous electrocardiograms (ECGs) in sinus rhythm were normal with no evidence of pre-excitation and a normal QT interval. The heart rate was 160 beats $\min ^{-1}$ and the blood pressure was $85 / 60 \mathrm{mmHg}$. The physical examination was otherwise unremarkable. A 12-lead
ECG demonstrated a regular narrow-complex tachycardia conducted with right bundle branch block morphology. Vagotonic manoeuvres were unhelpful and, therefore, adenosine was administered as a 3-mgh followed by a $6-\mathrm{mg}$ intravenous bolus. A continuous lead II rhythm strip of the events which followed the 6-mg bolus is shown (Fig. 1). Thirteen seconds after injection, four beats of non-sustained broad-complex tachycardia (suggestive of VT) were observed followed by a period of electrical instability over the next $12 \mathrm{~s}$. Sinus rhythm was restored without further complication and a subsequent 12-lead ECG was normal. Plasma electrolytes, cardiac enzymes and the results of thyroid function tests were normal. Transthoracic echocardiography was also normal but an electrophysiological study demonstrated the presence of a retrograde partial AV nodal bypass tract which was treated by catheter radiofrequency ablation.

\section{DISCUSSION}

With only a single-lead ECG recording it is not possible to unequivocally diagnose the non-sustained broad-complex tachycardia as ventricular tachycardia, the other possibility being acceleration of the re-entrant tachycardia with aberrant conduction. Nevertheless, two previous reports of serious ventricular arrhythmias (torsades de pointes in a patient with a long QT interval ${ }^{2}$ and non-sustained polymorphic $\mathrm{VT}^{3}$ ) suggest that these are an occasional adverse effect of adenosine injection. The Committee on Safety of Medicines has received four other reports of ventricular arrhythmia following adenosine: one of torsades de pointes leading to ventricular fibrillation, one of ventricular ectopics and two of ventricular tachycardia (Committee on Safety of Medicines, personal communication). Other tachyarrhythmias including delayed sinus tachycardia, ventricular extrasystoles ${ }^{4}$ and a paradoxical enhancement of AV nodal conduction ${ }^{5}$ have also been observed. Adenosine has a number of electrophy- 
Adenosine-induced tachycardia (a)

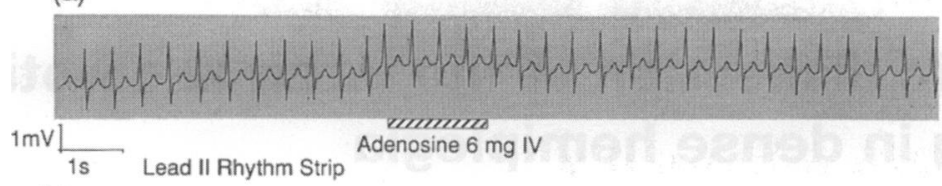

(b)

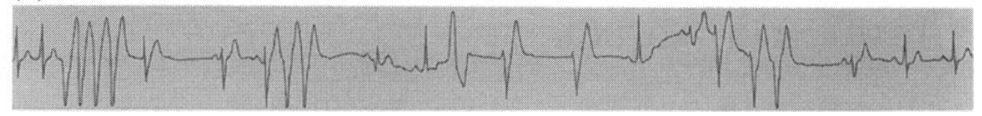

Fig. 1. Continuous lead II rhythm strip demonstrating (a) the regular narrow-complex tachycardia treated with intravenous adenosine and (b) the development of four beats of broad-complex tachydardia after adenosine injection. A period of electrical instability over the next $12 \mathrm{~s}$ is followed the restoration of sinus rhythm. siological effects including hyperpolarization of atrial myocytes, reduction in the diastolic depolarization of the pacemaker cells of the sinoatrial node and depression of conduction through the ' $\mathrm{N}$ ' cells of the AV node (the basis of its AV nodal blockade)'. Although it appears to have no direct effect on ventricular myocytes, adenosine has the potential to enhance cardiac excitability by activating the afferent arm of sympathetic autonomic reflexes, probably by stimulating chemoreceptors in the aortic arch. This indirect action provides a hypothetical basis for the observed adverse effect.

Vagotonic procedures (including the Valsalva manoeuvre, carotid sinus massage and cold water head immersion) remain the first-line treatment for paroxysmal re-entrant supraventricular tachycardias in the accident and emergency department. If these fail, adenosine should be administered but with continuous ECG monitoring and with the facilities for direct current cardioversion available. Clinicians should be alert to the possibility of tachy-as well as bradyarrhythmias following adenosine injection.

\section{REFERENCES}

1. Camm A.J. \& Garratt C.J. (1991) Adenosine and supraventricular tachycardia. New England Journal of Medicine 325, 1621-1629.

2. Wesley R.C. \& Turnquest P. (1992) Torsades de pointes after intravenous adenosine in the presence of the long QT syndrome. American Heart Journal 123, 794-796.

3. Romer M. \& Candinas R. (1994) Adenosine-induced nonsustained polymorphic ventricular tachycardia. European Heart Journal 15, 281-282.

4. Rankin A.C. Oldroyd K.G., Chong E., Rae A.P. \& Cobbe S.M. (1989) Value and limitations of adenosine in the diagnosis and treatment of broad complex tachycardia. British Heart Journal 62, 195-203.

5. Brodsky M.A., Allen B.J., Grimes J.A. \& Gold C. (1994) Enhanced atrioventricular conduction during atrial flutter after intravenous adenosine. New England Journal of Medicine 330, 288-289. 